\title{
Gestational Diabetes Mellitus (GDM) in the Republic of Kosovo: a Retrospective Pilot Study.
}

\author{
Armond Daci', Brikene Elshani², Giangiacomo Beretta ${ }^{3}$ \\ Department of Pharmacology, Faculty of Medicine, University of Prishtina, Prishtina, Republic of Kosovo ${ }^{1}$ \\ Department of Pharmaceutical Sciences, University of Milan, Milan, Italy ${ }^{2}$ \\ Gynecological Clinic of Kosovo (Qendra Klinike Universitare e Kosovës, QKUK), University Clinical Center of Kosovo, Prishtina, \\ Republic of Kosovo ${ }^{3}$
}

D) ackground: GDM is a condition in which women without previously diagnosed diabetes exhibit high blood glucose levels during pregnancy. Pregnancy causes some insulin resistance in all women, but only a few develop GDM. Objective: To test the hypothesis that women with GDM have impaired regulation of blood iron storage and transport, decreased renal function due to decreased glomerular filtration rate and occurrence of urinary tract infection (UTI). Study design and Methods: Incidence of blood iron storage was investigated in $n=30$ pregnant kosovar women with GDM after mild of pregnancy and in n=30 pregnant women without GDM (years 20102012). Results and Discussion: Baby weights, both systolic and diastolic BP, creatinine, albumin, lymphocytes, monocytes, WBC and granulocytes in both groups were within their normal ranges in both groups. Compared to control group, glucose was higher in women with GDM (mean \pm SD: $7.43 \pm 2.23 \mathrm{mg} / \mathrm{dL}$ vs. $4.33 \pm 0.63 \mathrm{mg} / \mathrm{dL} ; \mathrm{P}<0.001$ ). Women with GDM had also higher RBC (mean \pm SD: $4.4 \pm 0.8 \%$ vs. $3.8 \pm 0.3 \% ; \mathrm{P}<0.005)$ and HGB (mean \pm SD: $13.0 \pm 3.2 \mathrm{~g} / \mathrm{dL}$ vs. $11.2 \pm 1.4 \mathrm{mg} / \mathrm{dL}$; $\mathrm{P}<0.05$ ), and decreased renal functionality (MDRD-GFR: $92.8 \pm 25.8 \mathrm{~g} / \mathrm{dL}$ vs. $108.2 \pm 38.2 \mathrm{~g} / \mathrm{dL}$; $\mathrm{P}<.05)$.Conclusion: There is a potential association between iron status and GDM. The role of iron from diet and/or from supplementation in GDM pathogenesis needs still to be examined. In addition we have observed a decrease of glomerular filtration rate in women with GDM. Due to the lack of studies on the relationships between GDM and UTI, and to the retrospective design of the present investigation, it is difficult to establish whether UTI may be a GDM causal factor or a consequence of GDM symptoms, signs and/or of its correlated pathologies. Key words: Gestational diabetes, preeclampsia, blood iron regulation, urinary tract infections, oxidative stress.

Corresponding author: Armond Daci, MS, Msc. Faculty of medicine, University of Prishtina. Phone: +38138512929; cell: +37744418385; E-mail: armondd@hotmail.com.

\section{INTRODUCTION}

GDM is a condition in which pregnant women without previously diagnosed diabetes begin exhibiting high blood glucose levels during pregnancy, accounting for the $\sim 90 \%$ of all pregnancies complicated by diabetes (1). GDM affects around the $7 \%$ of all pregnancies and, depending on the population sample and diagnostic criteria, its prevalence may range from 1 to $14 \%(2)$. from south Asia, overweight, obesity or severe obesity increases the risk by a factor 2.1,3.6 and 8.6, respectively, a previous pregnancy which resulted in a child with a high birth which resulted in a child with a high birth weight $\left(>90^{\text {th }}\right.$ centile, or $>4000 \mathrm{~g}$ ( $8 \mathrm{lbs} 12.8 \mathrm{oz}$ ), previous poor obstetric history $(3,4)$. In addition to this, statistic show a double risk of GDM in smokers (5). Babies born from mothers affected by GDM are typically at increased risk of macrosomia $(6,7,8)$. And of additional problems such as the development of childhood obesity and of type 2 diabetes later in life. Women with GDM are at a significantly higher risk of developing preeclampsia or hypertension after pregnancy compared to healthy subjects (9), probably due to presence of preexisting common risk factors for both GDM and hypertension (10). Other studies have shown that GDM may be also associated to increased blood iron storage and transport $(11,12)$.In addition, different studies on the prevalence of urinary tract infections (UTI), symptomatic and/or asymptomatic bacteriuria in different populations of female diabetic patients reported contrasting results, showing that they can be either increased (13); or unchanged to this diabetic complication compared to non-diabetic subjects (14).

Hence, the aim of this study was (a) to furnish a preliminary description of the pathophysiological conditions of two selected population of Kosovar women with and without GDM, and (b) to evaluate the 
effects of diabetes on the impairment of blood iron physiological control, blood pressure and renal functionality status, and susceptibility to develop UTI.

\section{EXPERIMENTAL STUDY DESIGN AND METHODS}

Patients, pregnant women $(n=60)$ were recruited from the Obstetric-Gynecologic Clinic of Prishtina (QKUK, Republic of Kosovo) during the years 2010/2012 and divided in two groups on the basis of diagnosis: GDM group $(n=30)$ and control group (CTR, total $n=30$ without GDM, of which $n=18$ had normal clinical pregnancy status and $n=12$ treated for preeclampsia). All women gave delivery by Cesarean delivery exception for 6 patients for which delivery was stimulated by oxitocin administration. Patients were followed-up from 2010 to 2012. The measurements of blood parameters glycaemia, creatinine, albumin, lymphocytes, monocytes, granulocytes, white blood cells, red blood cells, haemoglobin, Glomerular filtration rate (GFR) was estimated using the modification of diet in renal disease (MDRD) algorithm [15]. Urine analysis with microscopy epithelial cells, leukocytes, bacteria, oxalate crystals and erythrocytes, as well as histological examination were carried out using standard assay procedures of laboratory of Biochemistry of the University Clinical Center of Kosovo (UCCK). The presence/absence of vaginal infections was established using standard microbiological protocols in institute of microbiology in the University Clinical Center of Kosovo (UCCK). Vaginal wet mount was observed with microscopy by placing the specimen on a glass slide and mixing with a salt solution.

Continuous clinical variables were compared by two-tailed pairwise Student's $t$ test for independent measurements with Bonferroni's correction for multiple comparisons and discrete variables by Fisher's exact test. Computations were conducted on original data and on $\log$ transformed data to reduce the effect of skewness. Minimal level of significance of the difference was set at $\mathrm{P}<0.05$. The data matrices for computations were built with an OpenOffice Calc Spreadsheet for Linux (v. 3.2.1), and all computations were done with R-commander GUI for R (v. 1.5-6) [16].

\section{RESULTS AND DISCUSSION}

In Table 1 and Table 2 are reported means, standard deviations and quintiles for the monitored physiological parameters (weights of babies, weeks of gestation at clinical visit, systolic and diastolic BP), blood biochemical parameters (creatinine, glucose, albumin, hemoglobin), blood cells profile (lymphocytes, monocytes, red blood cells (RBC), white blood cells (WBC) and granulocytes), and renal functionality determined in pregnant women with and without GDM respectively.

Gestation duration was lower than the full term of 40 weeks for normal delivery in both groups. The gestation time before delivery was 2.7 weeks shorter in group II (35.8 \pm 2.5 weeks) compared to group I ( $38.5 \pm 2.2$ weeks, $\mathrm{P}<0.001)$. Mean values of babies weight, and mothers' systolic and diastolic BP, creatinine, albumin, WBC and granulocytes in both groups were within their normal ranges, with no significant difference between the two groups $(P>0.05)$. However, for both groups, women falling into the two highest quintiles (IV and V) showed systolic and diastolic BP higher than reference values. In both groups, lymphocytes and monocytes mean values and quintiles were significantly elevated in respect to the matched reference values for the CTR group (17). Probably resulting from the presence of infections in both groups (see paragraph 3.2). As expected, blood glucose was higher in group I (mean \pm SD: $7.43 \pm 2.23 \mathrm{mg} / \mathrm{dL}$ vs. $4.33 \pm 0.63$ $\mathrm{mg} / \mathrm{dL} ; \mathrm{P}<0.001)$, on account of their diabetic status. Women in this group had also higher RBC (mean \pm SD: $4.4 \pm 0.8 \%$ vs. $3.8 \pm 0.3 \%$; $<<0.005$ ) and HGB (mean $\pm \mathrm{SD}: 13.0 \pm 3.2 \mathrm{~g} / \mathrm{dL}$ vs. $11.2 \pm 1.4 \mathrm{mg} /$ $\mathrm{dL} ; \mathrm{P}<0.05)$. Women with GD showed decreased renal functionality, as evidenced by the significant decrease of their mean GFR value in respect to that of women with preeclampsia (MDRD-GFR: $92.8 \pm$ $25.8 \mathrm{~g} / \mathrm{dL}$ vs. $108.2 \pm 38.2 \mathrm{~g} / \mathrm{dL} ; \mathrm{P}<0.05)$.

The high concentration of mitochondria in placenta, one of the major sources of free radicals in living organism, may be a causal/concurrent condition in the development of GDM, due to the oxidative stress that may be induced by the impairment of their activity. In particular, iron, which is abundant in placenta, is one of the most important generators of highly reactive free radicals in both normal and pathological conditions (18). Our findings that blood iron, in terms of blood HGB and $\mathrm{RBC}$, is significantly elevated in pregnant women affected by GDM compared with women with preeclampsia (Hb: 13.0 $\pm 3.2 \mathrm{~g} / \mathrm{dL}$ vs.11.2 $\pm 1.4 \mathrm{~g} / \mathrm{dL} ; \mathrm{P}<.05)$ are in good agreement with those recently reported in a similar study by Afkhami-Ardekani et al. (11). In this study the concentrations of serum ferritin, iron, transferrin saturation and $\mathrm{HGB}, \mathrm{MCV}$, and $\mathrm{MCH}$ were significantly higher in Iranian pregnant women with GDM (Hb: $13.4 \pm 1.1 \mathrm{~g} /$ $\mathrm{dL}$ vs. $11.8 \pm 1.4 \mathrm{~g} / \mathrm{dL} ; \mathrm{P}<.001)$, indicating a potential association between increased iron status and GDM. A few years earlier,

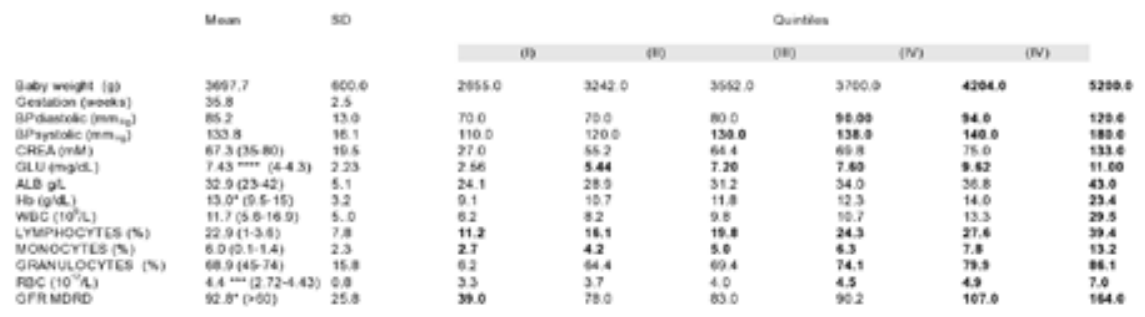

Table 1. Pregnant women with GD (normal ranges in brackets from ref 17. ${ }^{*} \mathrm{P}<0.05$; ${ }^{* \star *} \mathrm{P}<0.005$; ${ }^{* \star * * P}<0.001$ compared to CTR group. (tab.2). Values exceeding refernces values are in bold.

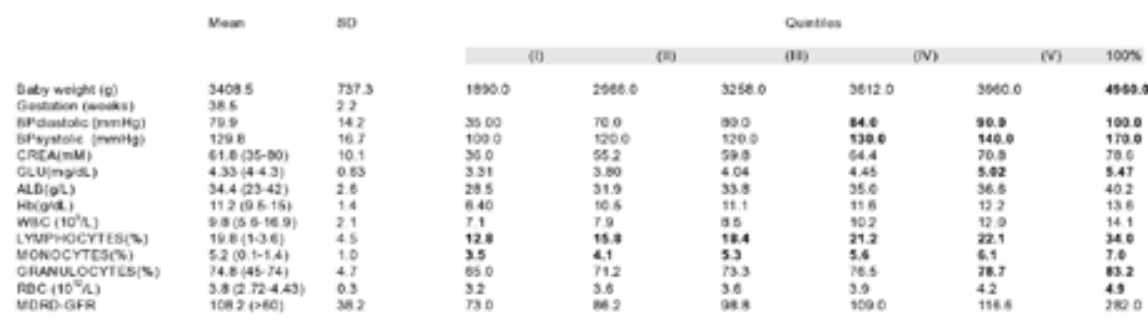

Table 2. Control pregnant women (normal ranges in brackets from ref. $17 .{ }^{\star} P<0.05$; ${ }^{\star \star \star} P<0.005$; ${ }^{\star \star \star \star} \mathrm{P}<0.001$ compared to CTR group. (tab.2). Values exceeding refernces values are in bold. 
Chan and colleagues concluded that increased iron storage and transport, (e.g. elevated serum ferritin), early in gestation are associated with an increased risk of GDM (12). The results of their study suggested that this association, at least in part, may be mediated by the maternal fat mass and obesity. Similar conclusions were reported in 2002 by Lao and colleagues working on a population of Chinese pregnant women (19). These authors found that high maternal hemoglobin (more than 13 $\mathrm{g} / \mathrm{dL}$ ) at the initial prenatal visit in a population of Chinese women is an independent risk factor for GDM. In another study the same authors found that maternal hepatitis B surface antigen (HBsAg) carriage could explain in part the association between increased serum ferritin concentration with $\mathrm{GD}$, and that the HBsAg carrier status is an independent risk factor for GDM (20).

The decrease of renal functionality we found in pregnant women affected by GDM $(115.3 \pm 16.0$ vs $101.3 \pm 18.5$ (Table 1 and Table 2) without significant increase of BP, indicated that this loss of renal functionality was probably associated to a glomerular damage still in an early stage of development. By contrast, as shown in Figure 1, the loss of renal functionality observed in pregnant women with GD was paralleled by a highly significant elevation of UTI compared to those affected by BP dysfunctions ( $100 \%$ vs. $13.3 \%$, $\mathrm{P}<0.001)$. These results were in good accordance with those from previous studies reporting that mothers affected by GDM are at higher risk of complications from UTI along with preeclampsia, hypertension, and Cesarean delivery (21). In particular, the predisposition to UTI in DM and GDM may result from several factors. Susceptibility to this kind of infections seems to increase with longer duration and greater severity of diabetes. As different studies have investigated the role of UTI in preeclampsia $(22,23,24,25$, 26). The role of UTI in GDM is poorly investigated and understood (27). Only few studies have shown that UTI caused by different bacteria are common in women with GD, and that their growth is probably due the favorable growth conditions induced by high blood and urine glucose.

\section{REFERENCES}

1. Setji T, Brown A, Feinglos M. Gestational Diabetes Mellitus. Clinical Diabetes. 2005; 30(1): 17-24.

2. American Diabetes Association. Gestational Diabe tes Mellitus. Diabetes Care. 2003; 26(1): 103-105. Ross G. Gestational diabetes. Aust Fam Physician. 2006; 35(6): 392-396.

4. Chu SY, Callaghan WM, Kim SY, Schmid CH, Lau J England LJ, Dietz PM. Maternal obesity and risk of gestational diabetes mellitus. Diabetes Care. 2007; 30(8): 2070-2076.

5. England LJ, Levine RJ, Qian C, Soule LM, Schisterman EF, Yu KF, Catalano PM. Glucose tolerance and risk of gestational diabetes mellitus in nulliparous women who smoke during pregnancy. Am J Epidemiol. 2004; 160(12): 1205-1213.

6. Preece R, Jovanovic L. New and future diabetes therapies: are they safe during pregnancy? J Matern Fetal Neonatal Med. 2002; 12(6): 365-375.

7. Langer O, Rodriguez DA, Xenakis EM, McFarland MB Berkus MD, Arrendondo F. Intensifi ed versus conventional management of gestational diabetes. Am J Obstet Gynecol. 1994; 170(4): 1036-1047.

8. Esakoff TF, Cheng YW, Sparks TN, Caughey AB. The association between birthweight $4000 \mathrm{~g}$ or greater and perinatal outcomes in patients with and without gestational diabetes mellitus. American Journal of Obstetrics \& Gynecology. 2009; 200(6): 672-674.

9. Tobias DK, Hu FB, Forman JP, Chavarro J, Zhang C. Increased Risk of Hypertension After Gestational Diabetes Mellitus: Findings from a large prospective cohort study. Diabetes Care. 2011; 34(7): 1582-1584.

10. Yogev Y, Xenakis EM, Langer O. The association between preeclampsia and the severity of gestational diabetes: the impact of glycemic control. Am J Obstet Gynecol. 2004; 191(5):1655-1660.

11. Afkhami-Ardekani M, Rashidi M. Iron status in women with and without gestational diabetes mellitus. J Diabetes Complications. 2009; 23(3):194-198

12. Chen X, Scholl TO, Stein TP. Association of elevated serum ferritin levels and the risk of gestational diabetes mellitus in pregnant women: The Camden study. Diabetes Care. 2006; 29(5): 1077-1082.

13. Cousins L. Pregnancy complications among diabetic women. Obstet Gynecol Surv. 1987; 42: 140-149.

14. Rizk DE, Mustafa N, Thomas L The prevalence of urinary tract infections in patients with gestational diabetes mellitus. Int Urogynecol J Pelvic Floor Dysfunct. 2001; 12(5): 317-321.

15. Levey AS, Bosch JP, Lewis JB, Greene T, Rogers N, Roth D. A more accurate method to estimate glomerular filtration rate from serum creatinine: a new prediction equation. Modification of Diet in Renal Disease Study Group. Ann Intern Med. 1999; 130(6): 461-470.

16. Fox J. The R Commander: A Basic-Statistics Graphical User Interface to R. J Stat Software. 2005; 14(9): 1-42.

7. Abbassi-Ghanavati M, Greer LG, Cunningham FG. Pregnancy and laboratory studies: a reference table for clinicians. Obstet Gynecol. 2009; 114(6): 1326-1331.

18. Halliwell B. Free radicals, antioxidants, and human disease curiosity, cause, or consequence? Lancet. 1994; 344(8924): 721-724.

19. Lao TT, Chan LY, Tam KF, Ho LF. Maternal hemoglobin and risk of gestational diabetes mellitus in Chinese women. Obstet Gynecol. 2002; 99(5): 807-812.

20. Lao TT, Tse KY, Chan LY, TAM KF, Ho LF. HBsAg carrier status and the association between gestational diabetes. Diabetes Care. 2003; 26(11): 3011-3016.

21. Khatun N, Latif SA, Uddin MM. Pregnancy associated complications of mothers with gestational diabetes mellitus. Mymensingh Med J. 2005; 14(2): 196-198.

22. Karmon A, Sheiner E. The relationship between urinary tract infection during pregnancy and preeclampsia. Arch Gynecol Obstet. 2008; 277(6): 479-481.

23. Mittendorf R, Lain KY, Williams MA, Walker CK Preeclampsia. A nested, case-control study of risk factors and their interactions. J Reprod Med. 1996; 41(7): 491-496.

24. Le J, Briggs GG, McKeown A, Bustillo G. Urinary tract infections during pregnancy. Ann Pharmacother. 2004; 238(10): 1692-1701.

25. Hsu CD, Witter FR. Urogenital infection in preeclampsia. Int J Gynaecol Obstet.1995; 49(3): 271-275.

26. Conde-Agudelo A, Villar J, Lindheimer M. Maternal infection and risk of preeclampsia: systematic review and metaanalysis. Am J Obstet Gynecol. 2008; 198(1): 7-22.

27. Chen SL, Jackson SL, Boyko EJ. Diabetes mellitus and urinary tract infection: epidemiology, pathogenesis and proposed studies in animal models. J Urol. 2009; 182(6): 51-56. 